\title{
New perspectives in glaucoma treatment
}

\begin{abstract}
Abbreviations: RGCs, retinal ganglion cells; FC, functional connectivity; MRI, magnetic resonance imaging

\section{Editorial}

Glaucoma is a neurodegenerative disease, characterized by a progressive optic disc cupping, a loss of retinal ganglion cells (RGCs) and a visual field damage, representing one of the leading causes of irreversible blindness worldwide. Even if, until today, it is considered "only" an ocular pathology, there are even more evidences that glaucoma involved the entire central nervous system or at least the functional connectivity (FC) of the visual network. A recent study (Structural and Functional Brain Changes beyond Visual System in Patients with Advanced Glaucoma Frezzotti P. et coll) shows, by using several type of Magnetic Resonance Imaging (MRI), presence of structural and functional changes in patients with advanced POAG, involving well beyond the visual system, suggesting that POAG can be considered a vision disorder belonging to brain's neurodegenerative disorders.
\end{abstract}

Like for the other neurodegenerative conditions, glaucoma research efforts is looking for a way to prevent cells loss by intervening in the neuronal death pathways. Among the known risk factors, elevated IOP is the most studied one and nowadays represents the only recognized therapeutic target 6 even if most patients with elevated IOP never develop glaucoma. Major limitations to an exclusive lowering IOP approach are:

i. There are many patients with an apparently controlled low IOP who continue to worsen.

ii. Many glaucoma patients do not have a statically elevated pressure (ie, low-tension glaucoma).

Neuroprotection found, so, his importance on his potentiality of increasing surviving cell capacity and preventing further structural and functional damage of the nervous structures involved through an interruption of the pathologic mechanism. Though pathogenetic primary process of glaucoma have not yet been fully cleared up, several key processes have been identified: mechanical compression, 8 ischemia, oxidative stress, neurotrophic growth factor deprivation intracellular calcium toxicity, activation of autoimmunity and glutamate neurotoxicity.

Some mechanisms leading to cell death are at the base of cellular normal function; that is how many drugs, as excitotoxicity-blocking agents, working in theory, have showed many intolerable side effects. Among these drugs, Memantine is seemed the most interesting also due to his use in treating other neurodegenerative pathologies such as the Alzheimer's disease14 or HIV related dementia.

Memantine works by binding $\mathrm{Mg}^{2+}$ modulating site on NMDA receptor bond permitted by their pathologic overactivity; that is how Memantine is ineffective in blocking physiological NMDA receptor activity. Despite this premises there was no significant evidences in vivo of his effect on stopping glaucoma progression. Obtaining an intracellular calcium excess is a key point for apoptotic mechanism
Volume 4 Issue 4 - 2016

\author{
Gianluca Scuderi,Antonio Greco \\ Department of NESMOS (Neurosciences, Mental Health and \\ Sensory Organs), "Sapienza” University of Rome, Italy
}

\begin{abstract}
Correspondence: Antonio Greco, NESMOS (Neurosciences, Mental Health and Sensory Organs) Department, School of Medicine and Psychology, "Sapienza" University of Rome, Sant'Andrea Hospital, Responsabile centro Glaucoma e Cornea, Via di Grottarossa 1035-39, 00189 Roma, Italy, Tel+390633775035, Email antonio.greco89@gmail.com
\end{abstract}

Received: June 17, 2016 | Published: June 20, 2016

trigger and NMDA receptor activity is central on his rise up. By this observation is born the suggestion that $\mathrm{Ca} 2+$ channel blockers are neuroprotective in glaucoma. Among $\mathrm{Ca}^{2+}$ channel blockers, dihydropyridinic molecules are the more promising grace to their important antioxidant properties. According to a recent 3-year study (Koseki N Ophthalmology 2008) orally administered nilvadipinean dihydropyridinic $\mathrm{Ca}^{2+}$ channel blocker-slightly slowed down visual field degeneration and maintained the optic disc rim of patients with normal low-tension IOP open-angle glaucoma. Besides conventional pharmaceutical products, it has been recognized an enormous importance to nutraceuticals. Employing nutraceuticals belongs to a more holistic approach to pathology itself: in fact it seems meaningful-in addition to standard glaucoma therapy-trying to improve patient's general quality of life, paying attention to patient's emotional condition knowing that it is plausible that psychological stress may have an influence on IOP and on pathology's worsening.

Curcumin, for example, is one of them. It belongs to the ginger family (Zingiberaceae) and has showed antioxidant, antitumor, chemopreventive, and anti-inflammatory action during acute/chronic inflammation, both in clinical and in experimental models. These properties are due to the lowering effect on mediators level like NF-kB, prostaglandin E-2, interleukins (TNF-a) or the reduction of genes expression, like IkBa gene or cyclooxygenase-2 one. Yan-Kun and colleagues recently (2014) tried to evaluate curcumin's effects both in vitro - simulating an oxidative stress condition-and in vivo in a chronic high IOP rat model. Their observed in vitro an increasing of cell viability and a decreasing of intracellular ROS and apoptosis significantly, while in vivo, the curcumin-treated group, has shown a significative reduction of cells loss besides a Caspase 3, Cytochrome $\mathrm{c}$ and BAX lowered levels.

Another nutraceutical studied for his neuromodulatory effects is homotaurine. His neuroprotective effects would seems to result from his GABA-A receptor agonist activity and inhibitor NMDA receptor inappropriate-activity capacity. In an study published in 2015 (Russo R 2015), an association between homotaurine, forskolin and L-carnosine it's tested. It seems that in the presence of homotaurine and L-carnosine, a more permissive environment for the neuroprotective effects exerted by forskolin is generated, increasing 
RGCs' responsiveness to endogenous trophic molecules, as BDNF and CTNF, that are transiently and early up regulated as part of the retinal defense responses.

Flavonoids are a class of plant and fungus secondary metabolites, ubiquitous in nature, particularly abundant in parsley, onions, blueberries and other berries, black tea, green tea dark chocolate (with a cocoa content of $70 \%$ or greater) [further information on dietary sources of flavonoids can be obtained from the US Department of Agriculture flavonoid database]. Even if there are conflicting evidences, it seems that these compounds have effects on reducing damage from oxidative stress. A recent meta-analysis (Shaheen $\mathrm{P}$ 2015) analyzes the informations available about that, concluding that flavonoids have a significant favorable effect on visual field in patients with glaucoma and ocular hypertension. Subgroup analysis illustrated that flavonoid intake is more favorable over a relatively short time frame in those with poorer visual field scores at baseline.

The list of substances could be infinite, certainly in future hypotensive and neuroprotective strategies will be investigated more and more together. The hope is that by focusing on neurological protection and enhancement capacity of some substances, we will be able not only to stop worsening of the disease, but also, in a particularly positive perspective, to achieve a turnaround.

\section{Acknowledgments}

None.

\section{Conflicts of interest}

The author is Scientific Advisor to Farkas Eye Black, Inc and is on the Board of Directors for Farkas Eye Black, Inc which was founded in 2000; any and all compensation has been donated to Dr. DeBroff's nonprofit organization: Distressed Children and Infants International.

\section{Funding}

None. 